\title{
Das Anti-Jo-1-Syndrom - eine Sonderform der Myositis mit interstitieller Lungenerkrankung
}

\author{
The Anti-Jo-1 Syndrome - A Specific Form of Myositis with Interstitial Lung \\ Disease
}

Autoren

Institute
A. Häussermann ${ }^{1}$, A. Gillissen ${ }^{1}$, W. Seidel ${ }^{2}$

Robert-Koch-Klinik, Thoraxzentrum des Klinikums St. Georg, Leipzig

Medizinische Klinik West des Klinikums St. Georg, FB Rheumatologie, Leipzig eingereicht 6.1 .2010 akzeptiert nach Revision 25.2.2010

\section{Bibliografie}

DOI http://dx.doi.org/ 10.1055/s-0029-1244054 Online-Publikation: 12. 4. 2010 Pneumologie 2010; 64: 496-503 @ Georg Thieme Verlag KG Stuttgart · New York ISSN 0934-8387

Korrespondenzadresse Dr. med. A. Häussermann Robert-Koch-Klinik Thoraxzentrum des Klinikums St. Georg Nikolai-Rumjanzew-Str. 100 04207 Leipzig anja.haeussermann@gmx.de

\section{Zusammenfassung \\ $\nabla$}

Erkrankungen des Lungenparenchyms treten häufig im Rahmen von entzündlichen Systemerkrankungen auf. Anti-Synthetase-Antikörper, einschließlich der Anti-Jo-1-Antikörper (Antihistidyl-tRNA-Synthetase), werden bei $25-35 \%$ aller Patienten mit einer Myositis nachgewiesen, wobei das Auftreten von Anti-Jo-1-Antikörpern mit $80 \%$ am häufigsten vertreten ist und einen prädiktiven Faktor für die Entwicklung einer interstitiellen Lungenerkrankung (ILE) darstellt. Umgekehrt sind Anti-Jo-1-Antikörper jedoch sehr selten bei ILE. Beim Auftreten von Myositis, Dyspnoe, Fieber, Arthralgien, Mechanikerhänden und/oder einer Raynaud-Symptomatik muss daher differenzialdiagnostisch auch an das Anti-Jo-1-Syndrom gedacht werden. Weitere diagnostische Hinweise ergeben sich aus dem radiomorphologischen Befund im HRCT, dem histopathologischen Muster der Lungenbiopsie (VATS) und evtl. auch dem Nachweis IFN- $\gamma$-induzierbarer Chemokine. Die Therapie besteht aus einer Immunsuppression, die nach Fallkontrollstudien häufig zum Erfolg führt. Kontrollierte Studien sind allerdings rar. In dieser Arbeit wird das Anti-Jo-1-Syndrom in einer Übersicht vorgestellt und eine Kasuistik als Beispiel angeführt sowie die verfügbaren Therapieoptionen diskutiert.

\section{Einleitung}

$\nabla$

Interstitielle Lungenerkrankungen (ILE) sind eine heterogene Gruppe nichtinfektiöser, nichtmaligner Veränderungen der Lunge mit entzündlicher Zellinfiltration und interstitieller Fibrose. Seit der Erstbeschreibung der ILE im Zusammenhang mit Dermatomyositis (DM) durch Mills and Mathews 1956 ist die Assoziation von ILE mit Polymyositis/ Dermatomyositis (PM/DM) bekannt und in der Literatur in vielen Fallbeispielen beschrieben [1]. Gemeinsames Merkmal der unterschiedlichen

\section{Abstract \\ $\nabla$}

Interstitial lung disease (ILD) may be associated with systemic inflammatory disorders and autoantibody production. The development of ILD has been described in detail in patients with polymyositis and dermatomyositis. Anti-synthetase antibodies, including anti-Jo-1 antibodies (antihistidyl-tRNA syntase), are found in up to $35 \%$ of patients with myositis, $80 \%$ of which constitute anti-Jo- 1 antibodies. The anti-Jo- 1 syndrome characteristically presents with myositis, shortness of breath, fever, polyarthritis/arthralgia, mechanic's hands, dermatomyositis-like skin lesions, signs of a connective tissue disease and/or Raynaud's phenomenon. ILD is an early diagnostic sign and shows focal infiltrates and an acinar pattern in CT scan. Non-specific interstitial pneumonitis with T-lymphocytic infiltrates in lung histology (VATS) or elevated IFN- $\gamma$-inducible chemokines are further indicators for anti-Jo-1 syndrome. Corticosteroids eventually combined with an immunosuppressant drug are often required with reported beneficial effects, although not many therapeutic studies have been performed. Here we present a review of the current literature and a case report on anti-Jo-1 syndrome.

Formen der Myositis ist dabei die Muskelschwäche durch entzündliche Infiltrate der Skelettmuskulatur, wobei die genauen pathophysiologischen Mechanismen bisher unbekannt sind. Bei 60$80 \%$ der Patienten mit PM/DM können Antikörper gegen intrazelluläre nukleäre oder zytoplasmatische Zellbausteine nachgewiesen werden [2]. Am häufigsten finden sich darunter Anti-Jo-1-Antikörper, welche mit einer Sonderform der Myositis, dem Antisynthetase- oder Anti-Jo-1-Syndrom assoziiert sind [3]. Nishikai und Reichlin charakterisierten das Krankheitsbild erstmals 1980 bei 
dem Patienten John P., in dessen Serum Antisynthetaseantikörper nachgewiesen wurden [4]. Das klinische Bild des Anti-Jo-1Syndroms manifestiert sich im Auftreten von Myositis, ILE, Polyarthritis, Fieber, Raynaud-Symptomatik und Mechanikerhänden (Hyperkeratose, Hyperpigmentation und Fissuren an den palmaren Fingerseiten), wobei die klinische Ausprägung variieren kann [5,6]. Das Auftreten von ILE bei Patienten mit Myositis beeinflusst wesentlich deren Morbidität und Mortalität [7-11].

\section{Epidemiologie und Prävalenz}

Die Inzidenz der PM beträgt rund 30\% und der DM ca. 25\% [12]. Sie kommen weltweit vor und haben einen Erkrankungsgipfel in der Kindheit und einen weiteren zwischen dem 50. und 70. Lebensjahr $[12,13]$. Das weibliche Geschlecht scheint in allen Altersgruppen zu überwiegen. Eine ILE wird bei diesen Patienten mit einer Prävalenz von 23-65\% in der Literatur beschrieben $[14,15]$. Umwelteinflüsse für das Auftreten der Erkrankung werden diskutiert, insbesondere genetische Faktoren und virale Infektionen als Trigger für autoimmunologische Prozesse, da eine saisonale Häufung im Frühjahr bei Patienten mit Myositis auffällig ist [16-22].

Antisynthetase-Antikörper werden bei 25-35\% der Patienten mit Myositis gefunden, wobei in $80 \%$ der Fälle Anti-Jo-1-Antikörper nachweisbar sind [14,23-25,27,29]. Der stärkste Prädiktive Faktor für die Entwicklung einer ILE im Rahmen einer Myositis ist der Nachweis von Anti-Jo-1-Antikörpern (Anti-AminohistidyltRNA-Synthetase-Antikörper) [9,20,26]. Diese treten mit einer Prävalenz von $10-40 \%$ bei Patienten mit Polymyositis (PM) und $2-10 \%$ mit Dermatomyositis (DM) sowie in 3-8\% bei der Overlap-Myositis auf $[14,27]$. Andere Anti-Aminoacyl-tRNA-Synthetasen kommen bei Myositispatienten jeweils nur in $1-3 \%$ vor. Die Assoziation von Anti-Jo-1-Antikörpern mit weiteren myositisassoziierten Autoantikörpern beträgt ca. 60\% [15]. Neben dem Nachweis von Anti-Jo-1-Antikörpern ist das Alter bei Erstmanifestation von $>45$ Jahren ein prädiktiver Faktor für die Entwicklung einer ILE bei PM/DM $[28,30]$. ILE kommen auch bei anderen Kollagenosen mit Nachweis myositisassoziierter Antikörper vor, z. B. bei systemischem Lupus erythematodes (SLE), Rheumatoide Arthritis (RA), Progressiv Systemische Sklerose (PSS), Sjögren-Syndrom (SS) und einer Überlappung dieser Kollagenosen (SLE, PSS, RA, PM) im Rahmen der Mixed connective tissue disease (MCTD) bzw. Mischkollagenose. Daher sollte bei Myositis mit ILE sorgfältig nach einer Mischkollagenose (Overlap-Syndrom) gesucht werden (s. Tab. 1) [2,23,31,32,48].

\section{Ätiologie und Pathogenese \\ $\nabla$}

Die Pathogenese des Anti-Jo-1-Syndroms ist bisher ungeklärt. Durch immunhistochemische Untersuchungen von Muskelbiopsien konnten humorale und zellvermittelte Immunprozesse nachgewiesen werden, die zur Muskelfaserschädigung führen. Der charakteristische Befund zeigt bei PM endomysial lokalisierte $\mathrm{CD}^{+} \mathrm{T}$-Lymphozyten mit Invasion der nichtnekrotischen Muskelfasern und bei der DM vorwiegend perivaskulär und in den peri- bzw. innerfaszikulären Septen ohne vergleichbare Invasion, wobei der genaue Mechanismus der zellvermittelten Zytotoxizität nicht bekannt ist $[2,28,33,34]$. Myositisspezifische Antikörper richten sich direkt gegen Aminoacyl-Transfer RNA (tRNA) Synthetasen, d. h. Enzyme, die bei der Proteinbiosynthese für die
Tab. 1 Myositisassoziierte Antikörper [22, 30].

\begin{tabular}{|lll|}
\hline $\begin{array}{l}\text { Antikörper } \\
\text { Anti-PM-Scl }\end{array}$ & $\begin{array}{l}\text { Prävalenz } \\
8-10 \%\end{array}$ & $\begin{array}{l}\text { Vorkommen/Syndrome } \\
\text { PM/DM, PSS und Overlap; häufig } \\
\text { ILE u. Arthralgie }\end{array}$ \\
\hline Anti-Ku & $1-9 \%$ & $\begin{array}{l}\text { PM/DM-PSS-Overlap, SLE, PSS, SS } \\
\text { Overlap PM/DM mit SLE u. PSS } \\
\text { immer bei MCTD }\end{array}$ \\
\hline Anti-U1-RNP & 12 & $\begin{array}{l}\text { PM/DM-Overlap mit PSS, selten } \\
\text { SLE, gewöhnlich keine ILE }\end{array}$ \\
\hline Anti-Ro (SS-A) & $10 \%$ & PM/DM-Overlap mit SLE, SS \\
\hline Anti-La (SS-B) & $1 \%$ & PM/DM-Overlap mit SLE, SS \\
\hline Anti-SRP & $\leq 5 \%$ & $\begin{array}{l}\text { PM mit schwerer Myositis, häufig } \\
\text { kardiale Beteiligung }\end{array}$ \\
\hline Anti-Mi2 & $\leq 8 \%$ & klassische DM \\
\hline
\end{tabular}

Tab. 2 Prävalenz von Antisynthetase Antikörpern bei PM/DM [34].

$\begin{array}{lll}\begin{array}{l}\text { Anti-tRNA- } \\ \text { Synthetasen }\end{array} & \text { Antigen } & \text { Prävalenz } \\ \text { Jo-1 } & \text { Histidin-tRNA-Synthetase } & 15-20 \% \\ \text { PL-7 } & \text { Threonin-tRNA-Synthetase } & 5-10 \% \\ \text { PL-12 } & \text { Alanin-tRNA-Synthetase } & 5 \% \\ \text { EJ } & \text { Glycin-tRNA-Synthetase } & 5-10 \% \\ \text { OJ } & \text { Isoleucin-tRNA-Synthetase } & 5 \% \\ \text { KS } & \text { Asparaginin-tRNA-Synthetase } & 5 \% \\ \text { Zo } & \text { Phenylalanin-tRNA-Synthetase } & 1 \% \\ \text { YRS } & \text { Tyrosin-tRNA-Synthetase } & 1 \%\end{array}$

Anbindung der verschiedenen Aminosäuren an ihr entsprechendes tRNA-Molekül verantwortlich sind. Bisher wurden bei 8 von 20 Aminoacyl-tRNA-Synthetasen Autoantikörper beschrieben, die mit unterschiedlicher Krankheitsausprägung und Prognose bei PM/DM assoziiert sind (s. $\bullet$ Tab. 2) [35].

\section{Klinische Manifestation}

Der Krankheitsbeginn bei PM/DM tritt meist subakut ein mit Abgeschlagenheit, Myalgie und typischerweise symmetrischer proximaler Muskelschwäche, die von den Patienten häufig als Schwierigkeit bei der Verrichtung alltäglicher Aktivitäten beklagt wird, wie z.B. Haare kämmen, Treppen steigen. Die Gesichtsmuskulatur ist nicht betroffen, jedoch können Dyspnoe und Dysphagie bei Affektion der Atem- und Schlundmuskulatur auftreten. Bei pulmonaler Beteiligung kommen unspezifische Symptome wie Husten und Dyspnoe vor, sind jedoch nicht in jedem Fall vorhanden [25].

Patienten mit einem Anti-Synthetase-Syndrom weisen überdurchschnittlich häufig eine ILE (50-75\%), Myositis (77-90\%), Arthritis (50-94\%), Raynaud-Syndrom (62-93\%) und Fieber (87\%) auf, was als Symptomkomplex unter dem Begriff Anti-Synthetasen-Syndrom zusammengefasst wird [5,6,20,36-38]. Im Gegensatz zu den Mischkollagenosen findet sich beim Anti-Syntetase-Syndrom keine über das Raynaud-Syndrom hinausgehende sklerodermietypische Symptomatik. Ein echter Overlap ist bei myositisspezifischen Antikörpern selten (<6\%) [2].

Wie bei der DM werden auch beim Anti-Jo-1-Syndrom häufig „Mechanikerhände“ beschrieben mit verdickter rissiger Haut und dunklen Querfalten an der Palmarseite der Finger [20,39].

Die o.g. Symptome bei ILE können bis zu 2 Jahre vor der eigentlichen muskulären Manifestation auftreten [9,40,41]. Jedoch sind respiratorische Symptome nicht regelhaft zu finden, denn Hus- 
ten oder Dyspnoe können auch bei ausgeprägtem radiologischen Lungenbefund und Einschränkung bei der Lungenfunktionsprüfung fehlen. Patienten mit ILE bei idiopatisch inflammatorischer Myositis können hinsichtlich ihrer klinischen Symptomatik in drei Gruppen eingeteilt werden [42,43]:

- akutes Auftreten,

- langsam progressiver Verlauf

- asymptomatische Manifestation (ohne pulmonale Symptome, aber mit auffälligem Röntgenthorax und pathologischen Lungenfunktionstests)

Der Gelenkbefall bei PM kann den muskulären Beschwerden vorausgehen und in der Frühphase mit einer RA verwechselt werden. Die Gelenkbeschwerden sind jedoch im Vergleich zur RA nur gering ausgeprägt und von kurzer Dauer. Im Vordergrund stehen Arthralgien und leichte Schwellungen vorwiegend der Finger-, Hand- und Kniegelenke, die meistens mit Beginn der Glucokortikoidtherapie rasch wieder verschwinden. Auch Gelenkergüsse wurden beschrieben, erosive Veränderungen an den Gelenken kommen in der Regel jedoch nicht vor. Patienten mit Anti-Jo-1-Syndrom zeigen signifikant häufiger Gelenkdeformationen insbesondere der distalen Interphalangealgelenke und der Daumengelenke als Patienten ohne Anti-Jo-1-Antikörper $[44,45]$.

Neben muskulären Symptomen der PM/DM können auch andere Organmanifestationen gefunden werden, wobei sich eine Herzmuskelbeteiligung bei über $70 \%$ der Patienten durch Nachweis von Kinetikstörungen, Septumasynchronie ect. in der Echokardiografie sowie durch EKG-Veränderungen darstellt und für 25\% der tödlichen Krankheitsverläufe durch Herzinsuffizienz, dilatative Kardiomyopathie oder Rhythmusstörungen verantwortlich ist [46].

\section{Diagnostik}

Zur Diagnosestellung einer Myositis sind bis heute die Kriterien nach Bohan und Peter gebräuchlich (s. Tab. 3) sowie die erweiterten Klassifikationskriterien nach Tanimoto et al. [23,47-49]. Bei allen Patienten mit Myositis sollten neben Laboruntersuchung mit Antikörperbestimmung, Elektromyographie (EMG) und Muskelbiopsie zur frühzeitigen Feststellung einer ILE Lungenfunktionstests, Röntgenthoraxuntersuchung und ein HR-CT Thorax durchgeführt werden.

Tab. 3 Diagnosekriterien der PM/DM nach Bohan und Peter 1975 modifiziert nach Tanimoto 1995 [23,48]: DM mindestens eine Hautveränderung und vier andere Kriterien (Sensitivität 94\% und Spezifität 95\%); PM mindestens vier Kriterien ohne Hautveränderung (Sensitivität 99\%, Spezifität 95\%).

symmetrische proximale Muskelschwäche evtl. mit Schluck- und Atem-
störung
Erhöhung der muskulären Serumenzyme insbesondere CK, Aldolase,
ALAT, ASAT, LDH
myopathische EMG-Veränderung mit pathologischer Spontanaktivität
bioptisch myositisches Gewebssyndrom mit Muskelfaserde- und -rege-
nerationszeichen und entzündlichen Infiltraten, evtl. mit Faserinvasion
charakteristische Hautveränderung bei Dermatomyositis wie Erythem
über Gelenkstreckseiten, Grottonzeichen (Erythem über Streckseiten
der Fingergelenke), livide Oberlidschwellung
Muskelschmerz bei Druck oder spontan
positive Anti-Jo-1-Antikörper
nichtdestruierende Arthritis oder Arthralgien
systemische Entzündungszeichen mit Fieber über $37^{\circ} \mathrm{C}$ axillär, erhöhte
CRP-Werte oder erhöhte BSG von > 20 mm/h nach Westergren

\section{Lungenfunktionstest}

Die Ausprägung der pulmonalen Veränderung stellt sich in der Lungenfunktionsprüfung als funktionelle Restriktionsstörung mit einer möglichen Reduktion der Vitalkapazität (VC) und des funktionellen Residualvolumens und einer eventuell bedingten verminderten Ein-Sekundenkapazität (FEV1) bei normalem Tiffeneau-Index (FEV1:FVC) dar. Insbesondere die Diffusionskapazität ist bei ILE häufig eingeschränkt und ein wichtiger, wenn auch unspezifischer Parameter zur Diagnostik und Verlaufskontrolle der ILE. Jedoch gibt es auch differenzialdiagnostisch Erkrankungen mit eingeschränkter Diffusionskapazität, wie z.B. die pulmonale Hypertonie [14]. Die Lungenfunktionsprüfung ist nicht nur wichtig für die Objektivierung der pulmonalen Schädigung, sondern auch zur Beurteilung des Krankheitsverlaufes und Therapieeffektes. Ebenso sollten die Blutgasanalyse zur Bestimmung einer Hypoxämie in Ruhe und unter Belastung, z.B. im 6-MinutenGehtest zur Verlaufsbeurteilung herangezogen werden.

\section{Bildgebende Verfahren}

Das Röntgenthoraxbild zeigt meist nur unspezifische Veränderungen, ist aber zur Verlaufsbeurteilung der Erkrankung und Beobachtung pulmonaler Komplikationen hilfreich. Ein normaler Röntgenthorax wird bei $10 \%$ der Patienten mit histologisch nachgewiesener ILE gefunden [50].

Aussagekräftiger bei Verdacht auf eine ILE ist ein HR-CT des Thorax, besonders zur Unterscheidung zwischen akuter Entzündung und Fibrose. CT-morphologische Aspekte, wie z. B. Milchglasphänomen, irreguläre Linien, retikuläre Konsolidierung oder Honigwabenmuster korrelieren mit dem histologischen Muster und werden bezogen auf die Klassifikation der interstitiellen Pneumonien der American Thoracic Society und der European Respiratory Society eingeteilt. Häufig zeigt sich bei Myositis und ILE das CT-morphologische Bild einer NSIP (Nicht spezifische interstitielle Pneumonie), wobei der Nachweis eines Milchglasphänomens mit der Entzündungsaktivität korreliert [51 - 56].

Bei Patienten mit Myositis insbesondere mit Nachweis von Antisynthetase-Antikörpern sollte auch ohne das Auftreten pulmonaler Symptome ein CT durchgeführt werden, da asymptomatische Lungenbeteiligungen vorkommen können $[26,41,57,58]$.

\section{Interventionelle Verfahren}

In der bronchoalveolären Lavage (BAL) lässt sich eine subklinische Alveolitis nachweisen und können evtl. differenzialdiagnostisch andere Ursachen einer ILE ausgeschlossen werden. Die BAL ist jedoch nicht spezifisch und nur in Zusammenhang mit den anderen diagnostischen Parametern zu werten [59]. Eine lymphozytäre Alveolitis kann ebenso wie eine neutrophile Alveolitis mit erhöhter Eosinophilenzahl in der BAL nachgewiesen werden, wobei letztere als Marker für eine progrediente Erkrankung mit schlechterer Prognose gewertet wird [9,41,42,84].

Aufgrund erhöhtem Morbiditäts- und Mortalitätsrisiko gehört die chirurgische Lungenbiopsie bei Patienten mit ILE und Myositis nicht zum Standard. Jedoch kann durch das HR-CT die histologische Diagnose aufgrund des spezifischen radiologischen Musters häufig vorhersagt werden. Bei myositisassoziierter ILE finden sich häufig verschiedene histopathologische Muster, so dass eine chirurgische Lungenbiopsie hilfreich sein kann, um eine Aussage über die Prognose der Erkrankung treffen zu können. Bei Nachweis einer zellulären NSIP oder einer COP (Kryptogen organisierende Pneumonie), welche am häufigsten bei myositisassoziierter ILE zu finden ist, zeigt sich ein eher gutes Ansprechen auf eine Glucocortikoidtherapie, wohingegen eine DAD (Diffuser 
Alveolarschaden) mit der schlechtesten Prognose zu assoziieren ist $[9,60-63]$.

Die transbronchiale Lungenbiopsie ist zur Diagnosestellung nicht hilfreich, da die Gewebestücke zur histologischen Beurteilung oft zu klein sind. Allerdings hat sie einen hohen Wert z.B. zur Beurteilung opportunistischer Pilzinfektionen infolge der immunsuppressiven Therapie [60].

\section{Laboruntersuchung}

Bei über 95\% der Patienten sind in den aktiven Krankheitsstadien muskuläre Serumenzyme wie Creatinkinase (CK), Aldolase, Lactatdehydrogenase (LDH) erhöht. Die Erhöhung der GesamtCK korreliert auf Grund ihrer relativen Muskelspezifität gut mit der Krankheitsaktivität. Normale Gesamt-CK-Werte können aber im chronischen Stadium mit ausgeprägter Muskelatrophie vorkommen, da die CK-Serumaktivität auch von der vorhandenen Muskelmasse abhängig ist. Bei PM/DM ist nicht nur das skelettmuskeltypische Isoenzym CK-MM, sondern gelegentlich auch die kardiale CK-MB erhöht, ohne dass klinisch eine kardiale Mitbeteiligung objektivierbar ist [64]. Bei Patienten ohne Muskelbeschwerden kann eine Erhöhung der Aminotransferasen und LDH mit einer Lebererkrankung verwechselt werden, wenn nicht gleichzeitig die Gesamt-CK mitbestimmt wird. Das CRP ist häufig normal. Beim Auftreten einer ILE ist die frühe Antikörpertestung, z. B. mittels ELISA und Immunfluoreszenz-Test, empfohlen. Wichtige Differenzialdiagnosen der ILE, wie Kollagenosen, Vasculitiden und die idiopathische Lungenfibrose, lassen sich durch die Bestimmung von Autoantikörpern, wie ANA (Antinukleäre Antikörper), ENA (extrahierbare Kernantigene), Doppelstrang-DNAAntikörper, p-ANCA und c-ANCA sowie Rheumafaktor und CCP (cyclisches citrulliniertes Peptid) eingrenzen [66,67]. Werden erhöhte ENA bestimmt, ist eine weitere Differenzierung notwendig zum Nachweis von myositisassoziierten Antisynthetasen (s. - Tab. 1 u. 2). Diese treten bei $60-80 \%$ der PM/DM Patienten auf und sind oft früh und meist persistierend im Krankheitsverlauf nachweisbar [65].

Zusätzlich zum positiven Nachweis von Anti-Jo-1-Antikörpern kommen bei myositisassoziierter ILE Biomarker vor, die sich für die prognostische Beurteilung als nützlich erwiesen haben. Dazu gehört das KL-6 (Krebs von den Lungen 6), ein von Typ-IIPneumozyten und Bronchialepithelzellen exprimiertes Glycoprotein sowie das Serum Surfactant Protein D und das CK-19 (Cytokeratin 19), da diese bei ILE erhöht nachweisbar sind und negativ mit der VC und Dlco korrelieren. CK-19 ist bei myositisassoziierter ILD mit AIP höher als mit NSIP [68 - 72]. Für die routinemäBige Analyse werden diese Parameter jedoch bisher nicht angewendet.

\section{Therapie}

$\nabla$

Für die Therapie der ILE bei Myositis ist die Datenlage uneinheitlich, da oft kleine Fallzahlen oder sogar nur Kasuistiken existieren und selbst innerhalb von Studien keine klaren Aussagen gemacht werden, aufgrund nicht konstant angewandter Therapieregime, fehlender Kontrolle durch den Placebo-Arm der Studien und kaum vorhandener miteinander vergleichbarer Studien. Ein weithin verbreitetes, trotzdem wenig evidenzbasiertes Behandlungsregime bei Myositis mit und ohne ILE stellt die Kortikosteroidtherapie dar, obwohl der monotherapeutische Ansatz häufig nicht suffizient zur Verbesserung der ILE führt und eine immunsuppressive Kombinationstherapie mit z.B. Methotrexat, Cyclo-
Tab. 4 Kriterien zur Beurteilung einer erfolgreichen immunsuppressiven Therapie.

sinkende CK-Werte

sinkende weitere laborchemische Parameter wie LDH, Aldolase, ALAT,

ASAT

Rückgang des entzündlichen Muskelinfiltrates

Steigerung der Diffusionskapazität sowie der VC

Verbesserung der Belastungsfähigkeit im 6-min Gehtest oder der Ergometrie

regrediente streifige Zeichnung im Röntgenthorax und der entzünd-

lichen morphologischen Veränderungen im Thorax-CT

phosphamid, Azathioprin und Ciclosporin A empfohlen ist $[9,41,73-78]$. Initial erfolgt die tägliche hochdosierte Einmalgabe eines oralen Prednisolonpräparates mit $0,75-1 \mathrm{mg} / \mathrm{kg} \mathrm{KG}$ über 6-8 Wochen. Kommt es zu einer Normalisierung oder mindestens Reduktion der CK-Werte, kann eine schrittweise Reduktion der Prednisolontherapie erfolgen, bis zu einer Minimaldosis, die zur Stabilisierung der Erkrankung notwendig ist. Diese sollte, um Rückfälle zu vermeiden, nach Erreichen des stabilen Zustandes mit normalen muskulären Serumenzymen über 1,5 bis 2 Jahre beibehalten werden. Anschließend kann ein vorsichtiger Auslassversuch durchgeführt werden, wobei sich auch hier die Empfehlungen auf kleine Fallzahlen ohne evidenzbasierte Nachweise bezieht. Bei chronischen Verläufen mit ausgeprägter Muskelatrophie können jedoch normale CK-Werte gemessen werden, obwohl die Entzündung aktiv und behandlungsbedürftig ist (s. - Tab. 4).

Das Ansprechen der Kortisontherapie ist abhängig vom Grad der Entzündung und Fibrose, wobei das histologische Bild einer COP im Gegensatz zu den anderen Mustern prognostisch am günstigsten ist $[14,51-56,89]$.

Mehrere kleine Studien beschrieben eine Verbesserung der ILE sowie den Rückgang der Myositisaktivität durch die Kombination von Glucocortikoiden mit Azathioprin (Dosis 2-2,5 mg/ $/ \mathrm{kg} \mathrm{KG}$ / Tag) oder Methotrexat (7,5 und $25 \mathrm{mg} /$ Woche p.o.) und Cyclophosphamid. Als messbare Parameter zeigten sich eine Steigerung im 6-Min-Gehtest, rückläufige CK-Werte oder auch geringere Lymphozyteninfiltration in der Biopsie [79]. Da die Azathioprinwirkung erst verzögert (frühestens 2 Monate nach Therapiebeginn) einsetzt, ist eine Beurteilung des Effektes erst nach ca. 6 Monaten möglich. Der Effekt von Methotrexat tritt im Vergleich dazu meist früher ein. Bei unzureichender Wirksamkeit aufgrund unterschiedlicher Resorption kann eine teurere s.c. Verabreichung durchgeführt werden. Bei Myositis mit ILE oder anderer extramuskulärer Organbeteiligung wird Methotrexat bevorzugt mit Cyclophosphamid und einer geringen Steroiddosis eingesetzt und führte zu einer Verbesserung von Dyspnoe, Belastbarkeit und Muskelschwäche sowie Regredienz der ct-morphologischen Befunde $[41,80,81]$. Seltener kommen Ciclosporin oder Chlorambucil zum Einsatz, daher sind die Erfahrungen mit diesen Präparaten nur anhand kleinerer Fallserien belegt [82-85,98]. Unter Therapie mit Ciclosporin verbesserte sich ebenso wie bei Methotrexat die Lungenfunktion, die 6-Min-Gehstrecke, und es zeigte sich eine Verringerung der Myositisaktivität im MRT, jedoch war erst später ein Absinken der Serum-CK nachweisbar [98]. Tacrolimus, eine dem Ciclosporin vergleichbare Substanz, brachte bei Patienten mit ILD und Anti-Jo-1-Antikörpern und Prednisolon/Methotrexatineffektivität eine Besserung der Lungenfunktion und des radiologischen Befundes im CT [86-88]. Bei einigen Patienten mit therapierefraktärer Myositis wurden Therapieerfolge mit hochdosierten i.v. Immunglobulinen (IVIG) verzeichnet, wobei 


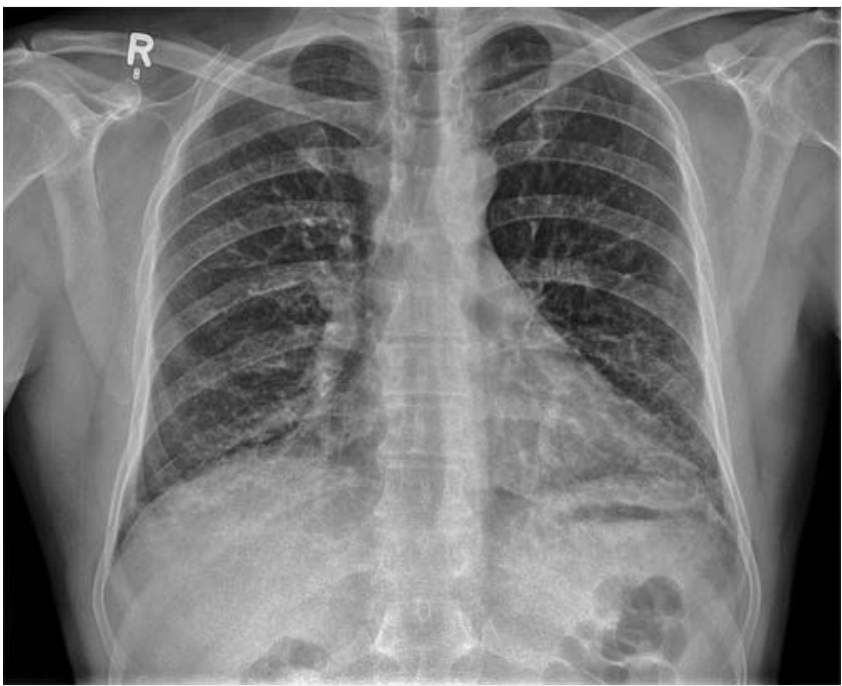

Abb. 1 Röntgen-Thorax a.p. mit streifiger Zeichnungsvermehrung der basalen Lungenfelder.

diese Therapie jedoch Nachteile durch die kurze Wirkdauer bei hohen Kosten darstellt $[85,102]$. In kürzlich erschienenen Fallserien wurden neue immunmodulierende Antikörper wie Rituximab bei schwerer ILE mit Myositis eingesetzt. Dadurch wurde eine Stabilisierung der klinischen Symptomatik und ein Rückgang der pathologischen ct-Befunde erreicht $[90,99]$. Der Einsatz von TNF-Antagonisten ist im Bereich der PM/DM und insbesondere mit ILE off label und bisher nicht publiziert [101].

Die meisten Patienten profitieren von einer immunsuppressiven Behandlung und erreichen bei PM/DM in ca. 30\% der Fälle eine Remission oder deutliche Besserung der Symptomatik (50\%). Jedoch liegt bei Nachweis von Anti-Jo-1-Antikörpern die 5-JahresÜberlebensrate unter 70\% und ist am geringsten bei Anti-SRPAntikörpern (25\%) aufgrund von kardiopulmonalen Komplikationen und Malignomen [21,22]. Anti-M2-Antikörper und PMScl-Antikörper und Anti-U1-RNP, wie häufig bei Mischkollagenosen nachweisbar, haben hingegen einen günstigen Krankheitsverlauf $[92,93]$.

Die Lungentransplantation kann als letzte therapeutische Option bei Patienten mit schwerer ILE im Endstadium ohne aktive Myositis und weitere Organbeteiligung erwogen werden, jedoch sind die Erfahrungen über das Outcome dieser Patienten gering.

\section{Prognose}

$\nabla$

Der natürliche Verlauf der PM zeigt in der Regel nach mehreren Jahren eine abnehmende Krankheitsaktivität. Spontane Remissionen scheinen nicht vorzukommen. Bei DM wurde ein rapid progressiver Verlauf häufiger als bei PM beschrieben [100]. Die meisten Todesfälle ereignen sich in den ersten 6 Monaten nach Diagnosestellung und sind auf kardiale und respiratorische Komplikationen oder Malignome zurückzuführen [7,9-11]. Die 1-, 3und 5-Jahres-Überlebensrate von Patienten mit PM/DM wurde mit $85,8-94,4 \%, 74,4-90,4 \%$ und $60,4-86,5 \%$ beschrieben $[9,62]$. Ein negativer prognostischer Faktor besteht bei Nachweis von ILE mit Hamman-Rich-Syndrom (akuter interstitieller Pneumonie), initial schwerer Diffusionsstörung unter 45\%, neutrophiler Alveolitis mit erhöhter Eosinophilenzellzahl sowie dem histopathologischen Nachweis von UIP und AIP $[9,41,43,60,94,95]$.

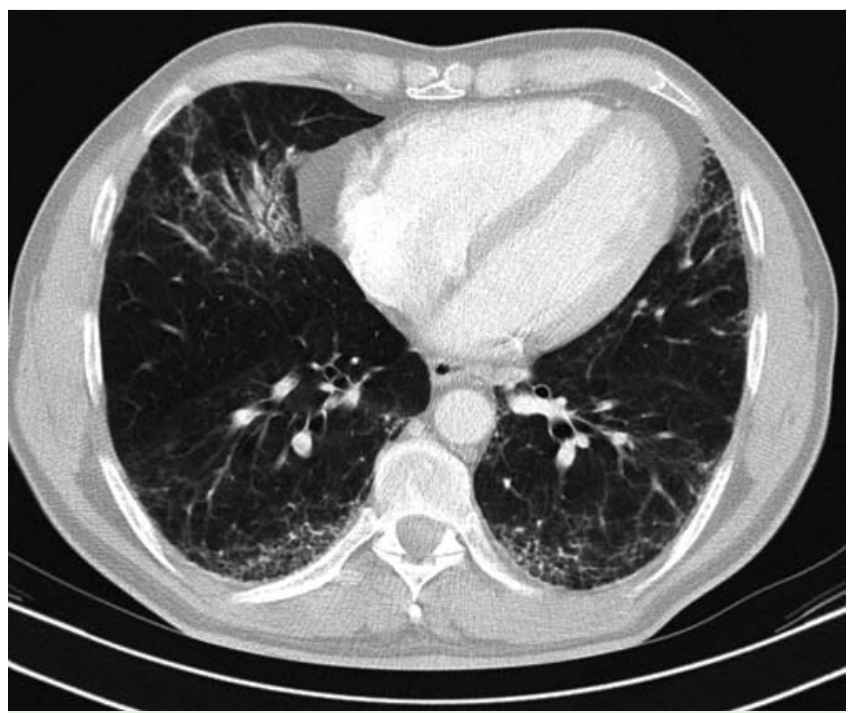

Abb. 2 CT-Thoraxschnitt mit pulmonaler Fibrose und Milchglasphänomen.

Die Assoziation von PM/DM und Malignomen wird in der Literatur mit einer Häufigkeit zwischen 7 und 34\% angegeben. Nach einer Metaanalyse an 1078 publizierten PM/DM Fällen wurden in der Zeitspanne von 5 Jahren vor bis 5 Jahren nach Erkrankungsbeginn mit durchschnittlich 15\% (PM ca. 10\% und DM ca. $20 \%$ ) gehäuft Mamma-, Ovar-, Lungen-, Magen- und Prostatakarzinom nachgewiesen [96]. Die pathogenetischen Vorgänge bei der Myositis-Tumor-Assoziation sind dabei völlig ungeklärt. PM/ DM mit ILE waren dabei nur selten mit einem malignen Tumor assoziiert im Gegensatz derer ohne ILE. Patienten mit myositisspezifischen Antikörpern wiesen eine negative Korrelation hinsichtlich der Tumorprävalenz auf [97]. Da das Anti-Jo-1-Syndrom eine Sonderform der Myositis darstellt, sollte auch hier sehr sorgfältig nach malignen Tumoren in den ersten 2 Jahren halbjährlichen, dann jährlichen Wiederholungsuntersuchungen in den ersten 5 Krankheitsjahren gesucht werden [2,96,97].

\section{Kasuistik}

$\nabla$

Ein 45-jähriger Mann wurde mit seit 2 Monaten bestehender progredienter Belastungsdyspnoe, Reizhusten ohne Auswurf und intermittierenden Fieberschüben stationär aufgenommen. Er litt zudem an einer Schwellung der Finger- und Handgelenke beidseits mit intermittierenden Schmerzen und Kribbelparästhesien. Eine Muskelschwäche war bisher nicht auffällig gewesen. Der Patient gab einen täglichen Konsum von 10 Zigaretten an (kumulativ ca. 15 pack years). Er ist als Lackierer tätig.

Bei der körperlichen Untersuchung bestanden bis auf eine auskultatorische ausgeprägte Sklerophonie basal beidseits über der Lunge keine Auffälligkeiten.

Lungenfunktionell ergab sich eine mäßiggradige Restriktion und mittelgradige Diffusionsstörung (FEV1 2,961=73,4\% v. Soll, VC $3,591=69,1 \%$ v. Soll, $T_{\text {LCO }} / 55 \%$ v. Soll). Die Blutgasanalyse ergab eine deutliche respiratorische Partialinsuffizienz (pO2 57 mmHg, pCO2 34 mmHg, pH 7,48).

Röntgenologisch zeigte sich eine streifige Zeichnungsvermehrung im Mittel- und Unterfeld der Lunge beidseits (s. Abb.1). Im CT-Thorax stellte sich eine pulmonale Fibrose mit Milchglasphänomen dar, die als UIP klassifiziert wurde (s. @ Abb. 2). 
Laborchemisch waren zunächst eine erhöhte CK $\left(22,27 \mu \mathrm{mol} / \mathrm{l}^{*} \mathrm{~s}\right)$, LDH $\left(7,51 \mu \mathrm{mol} / \mathrm{I}^{*} \mathrm{~s}\right), \operatorname{ALAT}\left(1,25 \mu \mathrm{mol} / \mathrm{l}^{*} \mathrm{~s}\right)$, ASAT $\left(1,35 \mu \mathrm{mol} / \mathrm{l}^{*} \mathrm{~s}\right)$ sowie CRP (5,1 mg/l) auffällig. Hämoglobin (8,6 mmol/l) und die absolute Lymphozytenzahl $(0,85 \mathrm{Gpt} / \mathrm{l})$ waren geringradig erniedrigt. Hinsichtlich der verschiedenen Differenzialdiagnosen bei pulmonaler Fibrose und der Kombination mit Gelenkbeschwerden und auffälligen Laborwerten wurden weitere Parameter bestimmt, wobei der ANA- und ENA-Screen positiv waren und die weitere Differenzierung den Nachweis von Jo-1-Antikörpern $(136 \mathrm{U} / \mathrm{ml})$ erbrachte.

Die Bronchoskopie war im Wesentlichen unauffällig, die transbronchiale Biopsie nicht verwertbar. In der BAL zeigte sich das Bild einer lymphozytären Alveolitis mit 31\% Lymphozyten und einem $\mathrm{CD}-\mathrm{T} 4^{+} / \mathrm{CD}-\mathrm{T} 8^{+}$Quotienten von 1:3,3. Zudem bestand auch eine geringe Eosinophilie (4\%) und Neutrophilie (7\%). Die weitere umfangreiche Diagnostik (Mikrobiologie, Echokardiografie, Abdomen-CT) erbrachte keinen pathologischen Befund.

Wegen des Nachweises von Anti-Jo-1-Antikörpern, dem Vorhandensein typischer Symptome wurde in Zusammenschau mit dem CT-Thoraxbefund und der Alveolitis ein Anti-Jo 1-Syndrom diagnostiziert.

Die Therapie wurde mit Prednisolon p.o. über 14 Tage begonnen $(0,7 \mathrm{mg} / \mathrm{kg} \mathrm{KG})$ mit einer anschließenden wöchentlichen Reduktion um $10 \mathrm{mg}$ bis auf eine Erhaltungsdosis von $10 \mathrm{mg} / \mathrm{Tag}$. Additiv erhielt der Patient eine Immunsuppression mit Methotrexat $15 \mathrm{mg}$ wöchentlich, Folsäure $5 \mathrm{mg}$ sowie Cyclosporin A $200 \mathrm{mg}$ morgens und abends.

Unter der Therapie kam es zu einem Abklingen der klinischen Symptome und zu einer Normalisierung der CK, ALAT und CRPWerte. Das weitere klinische und therapeutische Procedere erfolgte, wie oben angeführt.

\section{Interessenkonflikte}

Die Autoren geben an, dass kein Interessenkonflikt besteht.

\section{Literatur}

1 Mills ES, Mathews WH. Interstitial pneumonitis in dermatomyositis. JAMA 1956; 160: 1467-1470

2 Broich P, Jerusalem F. Polymyositis, Dermatomyositis und andere entzündliche Muskelerkrankungen. In: Miehle W, Fehr K, Schattenkirchner M, Tillmann K, Hrsg. Rheumatologie in Praxis und Klinik. 2. Aufl. Stuttgart: Thieme, 2000

3 Váncsa A, Csípö I, Németh J et al. Characteristics of interstitial lung disease in SS-A positive/Jo-1 positive inflammatory myopathy patients. Rheumatol Int 2009; 29: 989-994

4 Nishikai M, Reichlin M. Heterogeneity of precipitating antibodies in polymyositis and dermatomyositis. Characterization of the Jo- 1 antibody system. Arthitis Rheum 1980; 23: $881-888$

5 Yoshida S, Akizuki M, Mimori T et al. The precipitating antibody to an acidic nuclear protein antigen, the Jo-1, in connective tissue diseases: a marker for a subset of polymyositis with interstitial pulmonary fibrosis. Arthritis Rheum 1983; 26: 604-611

6 Marguerie C, Bunn CC, Beynon HL et al. Polymyositis, pulmonary fibrosis and autoantibodies to aminoacyl-tRNA synthetase enzymes. QJ Med 1990; 77: 1019-1038

7 Dickey BF, Myers AR. Pulmonary disease in polymyositis/dermatomyositis. Semin Arthritis Rheum 1984; 14: 60-76

8 Benbassat J, Gefel D, Larholt Ket al. Prognostic factors in polymyositis/ dermatomyositis: a computer-assisted analysis of ninety-two cases. Arthritis Rheum 1985; 28: 249-255

9 Marie I, Hachulla E, Cherin P et al. Interstitial lung disease in polymyositis and dermatomyositis. Arthritis Rheum 2002; 47: 614-622

10 Marie I, Hachulla E, Cherin P et al. Opportunistic infections in polymyositis and dermatomyositis. Arthritis Rheum 2005; 53: 155 - 165
11 Dankó K, Ponyi A, Constantin T et al. Long-term survival of patients with idiopathic inflammatory myopathies according to clinical features: a longitudinal study of 162 cases. Medicine 2004; 83: 35 - 42

12 Hengstman GJ, van Venrooij WJ, Vencovsky J et al. The relative prevalence of dermatomyositis and polymyositis in Europe exhibits a latitudinal gradient. Ann Rheum Dis 2000; 59: 141 - 142

13 Weitoft T. Occurrence of polymyositis in the county of Gavleborg, Sweden. Scand J Rheumatol 1997; 26: $104-106$

14 Fathi $M$, Lundberg IE. Interstitial lung disease in polymyositis and dermatomyositis. Current Opinion in Rheumatology 2005; 17: 701 706

15 Rutjes SA, Vree Egberts WT, Jongen P et al. Anti-Ro52 antibodies frequently co-occur with anti-Jo- 1 antibodies in sera from patients with idiopathic inflammatory myopathy. Clin Exp Immunol 1997; 109: $32-40$

16 Fox SA, Ward BK, Robbins PD et al. Inclusion body myositis: investigation of the mumps virus hypothesis by polymerase chain reaction. Muscle Nerve 1996; 19: 23-28

17 Leff RL, Love LA, Miller FW et al. Viruses in idiopathic inflammatory myopathies: absence of candidate viral genomes in muscle. Lancet 1992; 339: $1192-1195$

18 Friedman JM, Pachman LM, Maryjowski ML et al. Immunogenetic studies of juvenile dermatomyositis: HLADR antigen frequencies. Arthritis Rheum 1983; 26: 214-216

19 O'Hanlon TP, Carrick DM, Arnett FC et al. Immunogenetic risk and protective factors for the idiopathic inflammatory myopathies: distinct HLA-A, -B, -DRB1 allelic profiles and motifs define clinicopathologic groups in Caucasians. Medicine 2005; 84: $338-349$

20 Chinoy H, Salway F, Fertig $N$ et al. The UK Adult Onset Myositis Immunogenetic Collaboration (AOMIC). In adult onset myositis, the presence of interstitial lung disease and myositis specific/associated antibodies are governed by HLA class II haplotype, rather than by myositis subtype. Arthritis Res Ther 2006; 8: R13

21 Love LA, Leff RL, Fraser DD et al. A new approach to the classification of idiopathic inflammatory myopathy: myositis-specific autoantibodies define useful homogeneous patient groups. Medicine Baltimore 1991; 70: 360 - 374

22 Love LA, Miller FW. Noninfectious environmental agents associated with myopathies. Curr Opin Rheum 1993; 5: 712 - 718

23 Bohan A, Peter JB. Polymyositis and dermatomyositis. N Engl J Med 1975; 292: $344-347$

24 Targoff IN. Immune manifestation of inflammatory muscle disease. Dis Clin North Am 1994; 20: 857-880

25 Selva-O'Callaghan A, Labrador-Horrillo M, Solans-Laque R et al. Myositis-specific and myositis-associated antibodies in a series of eightyeight Mediterranean patients with idiopathic inflammatory myopathy. Arthritis Rheum 2006; 55: 791 - 798

26 Fathi M, Dastmalchi M, Rasmussen E et al. Interstitial lung disease, a common manifestation of newly diagnosed polymyositis and dermatomyositis. Ann Rheum Dis 2004; 63: 297-301

27 La Corte R, Lo Mo Naco A, Locaputo A et al. In patients with antisynthetase syndrome the occurrence of anti-Ro/SSA antibodies causes a more severe interstitial lung disease. Autoimmunity 2006; 39: 249 253

28 Takada K, Nagasaka K, Miyasaka N. Polymyositis/dermatomyositis and interstitial lung disease: a new therapeutic approach with $\mathrm{T}$ cell-specific immunosuppressants. Autoimmunity 2005; 38: $383-$ 392

29 Dalakas MC. Polymyositis and dermatomyositis. Lancet 2003; 362: 971-982

30 Chen I-J, Wu Y-J, Lin C-W et al. Interstitial lung disease in polymyositis and dermatomyositis. Clin Rheumatol 2009; 28: 639-646

31 Rider LG, Miller FW. Laboratory evaluation of the inflammatory myopathies. Clin Diagn Lab Immunol 1995; 2: 1 -9

32 Kalluri M, Sahn SA, Oddis CV et al. Clinical Profile of Anti-PL-12 Autoantibody. Chest 2009; 135: 1550-1556

33 Arahata K, Engel AG. Monoclonal antibody analysis of mononuclear cells in myopathies: I. Quantitation of subsets according to diagnosis and sites of accumulation and demonstration and counts of muscle fibers invaded by T cells. Ann Neurol 1984; 16: 193-208

34 Engel AG, Arahata K. Monoclonal antibody analysis of mononuclear cells in myopathies, II: Phenotypes of autoinvasive cells in polymyositis and inclusion body myositis. Ann Neurol 1984; 16: 209-215 
35 Mimori T, Imura Y, Nakashima R et al. Autoantibodies in idiopathic inflammatory myopathy: an update on clinical and pathophysiological significance. Curr Opin Rheumatol 2007; 19: 523 - 529

36 Bernstein RM, Morgan SH, Chapman J et al. Anti-Jo-1 antibody: a marker for myositis with interstitial lung disease. BMJ Clin Res Ed 1984; 289: 151 - 152

37 Schmidt WA, Wetzel W, Friedlander $R$ et al. Clinical and serological aspects of patients with anti-Jo-1 antibodies: an evolving spectrum of disease manifestations. Clin Rheumatol 2000; 19: 371 - 377

38 Hengstman GJ, van Engelen BG, van Venrooij WJ. Myositis-specific autoantibodies: changing insights in pathophysiology and clinical associations. Curr Opin Rheumatol 2004; 16: 692-699

39 Miller FW. Myositis-specific autoantibodies: touchstones for understanding the inflammatory myopathies. JAMA 1993; 270: $1846-$ 1849

40 Schwarz MI. The lung in polymyositis. Clin Chest Med 1998; 19 : 701 712

41 Schnabel A, Reuter M, Biederer J et al. Interstitial lung disease in polymyositis and dermatomyositis: clinical course and response to treatment. Semin Arthritis Rheum 2003; 32: 273 - 284

42 Frazier AR, Miller RD. Interstitial pneumonitis in association with polymyositis and dermatomyositis. Chest 1974; 65: $403-407$

43 Kang EH, Lee EB, Shin KC et al. Interstitial lung disease in patients with polymyositis, dermatomyositis and amyopathic dermatomyositis. Rheumatology Oxford 2005; 44: 1282 - 1286

44 Oddis CV, Medsger TA, Cooperstein LA. A subluxing arthropathy associated with the Anti-Jo-1 antibody in polymyositis/dermatomyositis. Arthritis Rheum 1990; 33: 1640-1645

45 Schumacher HR, Schimmer B, Gordon GV et al. Articular manifestations of polymyositis and dermatomyositis. Amer J Med 1979; 67: 287 292

46 Taylor AJ, Wortham DC, Burge JR, Rogan KM. The heart in polymyositis: a prospective evaluation of 26 patients. Clin Cardiol 1993; 16: $802-808$

47 Bohan A, Peter JB. Polymyositis and dermatomyositis. N Engl J Med 1975; 292: $403-407$

48 Tanimoto K, Nakano K, Kano $S$ et al. Classification criteria for polymyositis and dermatomyositis. J Rheumatol 1995; 22: 668-674

49 Fathi M, Lundberg IE, Tornling G. Pulmonary Complications of Polymyositis and Dermatomyositis. Semin Respir Crit Care Med 2007; 28: $451-458$

50 Epler GR, McLoud TC, Gaensler EA et al. Normal chest roentgenograms in chronic diffuse infiltrative lung disease. N Engl J Med 1978; 298: 934-939

51 Muller NL, Staples CA, Miller RR et al. Disease activity in idiopathic pulmonary fibrosis: CT and pathologic correlation. Radiology 1987; 165: 731 - 734

52 Muller NL, Miller RR, Webb WR et al. Fibrosing alveolitis: CT-pathologic correlation. Radiology 1986; 160: 585 - 588

53 Nishimura K, Kitaichi M, Izumi T et al. Usual interstitial pneumonia: histologic correlation with high-resolution CT. Radiology 1992; 182: $337-342$

54 Bonnefoy 0 , Ferretti G, Calaque 0 et al. Serial chest CT findings in interstitial lung disease associated with polymyositis-dermatomyositis. Eur J Radiol 2004; 49: 235 - 244

55 Lee CS, Chen TL, Tzen CY et al. Idiopathic inflammatory myopathy with diffuse alveolar damage. Clin Rheumatol 2002; 21: 391 - 396

56 Arakawa H, Yamada H, Kurihara Y et al. Nonspecific interstitial pneumonia associated with polymyositis and dermatomyositis: serial high-resolution CT findings and functional correlation. Chest 2003; 123: $1096-1103$

57 Remy-Jardin M, Giraud F, Remy J et al. Importance of ground-glass attenuation in chronic diffuse infiltrative lung disease: pathologic-CT correlation. Radiology 1993; 189: 693-698

58 Yang Y, Fujita J, Tokuda M et al. Chronological evaluation of the onset of histologically confirmed interstitial pneumonia associated with polymyositis/dermatomyositis. Intern Med 2002; 41: 1135-1141

59 Wallaert B, Hatron PY, Grosbois JM et al. Subclinical pulmonary involvement in collagenvascular diseases assessed by bronchoalveolar lavage: relationship between alveolitis and subsequent changes in lung function. Am Rev Respir Dis 1986; 133: 574-580

60 Tazelaar HD, Viggiano RW, Pickersgill J et al. Interstitial lung disease in polymyositis and dermatomyositis: clinical features and prognosis as correlated with histologic findings. Am Rev Respir Dis 1990; 141: $727-733$
61 Bjoraker JA, Ryu JH, Edwin MKet al. Prognostic significance of histopathologic subsets in idiopathic pulmonary fibrosis. Am J Respir Crit Care Med 1998; 157: 199-203

62 Douglas WW, Tazelaar HD, Hartman TE et al. Polymyositis-Dermatomyositis associated interstitial lung disease. Am J Respir Crit Care Med 2001; 164: $1182-1185$

63 Tansey D, Wells AU, Colby TV et al. Variations in histological patterns of interstitial pneumonia between connective tissue disorders and their relationship to prognosis. histopathology 2004; 44: 585 - 596

64 Bohlmeyer TJ, Wu AHB, Perryman MB. Evaluation of laboratory tests as a guide to diagnosis and therapy of myositis. Rheum Dis Clin N Amer 1994; 20: $240-250$

65 Bernstein RM. Autoantibodies in myositis. Baillières clin Neurol 1993; 2: $599-615$

66 Tillie-Leblond I, Wislez M, Valeyre D et al. Interstitial lung disease and anti-Jo-1 antibodies: difference between acute and gradual onset. Thorax 2008; 63: $53-59$

67 Zampieri S, Ghirardello A, Iaccarino L et al. Anti-jo-1 antibodies. Autoimmunity 2005; 38: $73-78$

68 Kubo M, Ihn H, Yamane K et al. Serum KL-6 in adult patients with polymyositis and dermatomyositis. Rheumatology 2000; 39: 632-636

69 Bandoh S, Fujita J, Ohtsuki Yet al. Sequential changes of KL-6 in sera of patients with interstitial pneumonia associated with polymyositis/ dermatomyositis. Ann Rheum Dis 2000; 59: 257-262

70 Ihn H, Asano Y, Kubo M et al. Clinical significance of serum surfactant protein D (SP-D) in patients with polymyositis/dermatomyositis: correlation with interstitial lung disease. Rheumatology 2002; 41: $1268-1272$

71 Fujita J, Dobashi N, Tokuda M et al. Elevation of cytokeratin 19 fragment in patients with interstitial pneumonia associated with polymyositis/dermatomyositis. J Rheumatol 1999; 26: 2377-2382

72 Gillissen A, Wiechmann V, Jürgens UR. Biomarker in pulmonary disease. Pneumologie 2009; 63: 439-450

73 Oddis CV. Current approach to the treatment of polymyositis and dermatomyositis. Curr Opin Rheumatol 2000; 12: 492 - 497

74 Nawata Y, Kurasawa K, Takabayashi $K$ et al. Corticosteroid resistant interstitial pneumonitis in polymyositis/dermatomyositis: prediction and treatment with cyclosporine. J Rheumatol 1999; 26: 1527 1533

75 Yoshida T, Koga H, Saitoh F et al. Pulse intravenous cyclophosphamide treatment for steroid-resistant interstitial pneumonitis associated with polymyositis. Intern Med 1999; 38: 733 - 738

76 Sakamoto N, Mukae H, Fujii T et al. Nonspecific interstitial pneumonia with poor prognosis associated with amyopathic dermatomyositis. Intern Med 2004; 43: 838-842

77 Kashiwabara K, Ota K. Rapidly progressive interstitial lung disease in a dermatomyositis patient with high levels of creatine phosphokinase, severe muscle symptoms and positive anti-Jo-1 antibody. Intern Med 2002; 41: $584-588$

78 Tomsic $M$, Sifrer F. Acute respiratory distress syndrome in a polymyositis patient with the anti-Jo-1 antibody. Wien Klin Wochenschr 2000; 112: $728-731$

79 Bunch TW. Prednisone and azathioprine for polymyositis. Arthritis Rheum $1981 ; 24: 45-48$

80 Schnabel A, Reuter M, Gross WL. Intravenous pulse cyclophosphamide in the treatment of interstitial lung disease due to collagen vascular diseases. Arthritis Rheum 1998; 41: 1215-1220

81 Yamasaki $Y$, Yamada $H$, Yamasaki $M$ et al. Intravenous cyclophosphamide therapy for progressive interstitial pneumonia in patients with polymyositis/dermatomyositis. Rheumatology 2007; 46: 124-130

82 Shinohara T, Hidaka T, Matsuki Y et al. Rapidly progressive interstitial lung disease associated with dermatomyositis responding to intravenous cyclophosphamide pulse therapy. Intern Med 1997; 36: 519 523

83 Dalakas MC. Polymyositis, dermatomyositis and inclusion body myositis. N Engl J Med 1991; 325: 1487 - 1498

84 Sauty A, Rochat T, Schoch OD et al. Pulmonary fibrosis with predominant CD8 lymphocytic alveolitis and anti-Jo-1 antibodies. Eur Respir J 1997; 10: 2907 - 2912

85 Sauty A, Rochat T, Schoch OD et al. A controlled trial of high-dose intravenous immune globulin infusions as treatment for dermatomyositis. N Engl J Med 1993; 329: 1993 - 2000

86 Selva-O'Callaghan A, Labrador-Horrillo M, Munoz-Gall X et al. Polymyositis/dermatomyositis-associated lung disease: analysis of a series of 81 patients. Lupus 2005; $14: 534-542$ 
87 Wilkes MR, Sereika SM, Fertig $N$ et al. Treatment of antisynthetaseassociated interstitial lung disease with tacrolimus. Arthritis Rheum 2005; 52: $2439-2446$

88 Oddis CV, Sciurba FC, Elmagd KA, Starzl TE. Tacrolimus in refractory polymyositis with interstitial lung disease. Lancet 1999; 353: 1762 1763

89 Dawson JK, Abernethy VE, Lynch MP. Effective treatment of anti Jo-1 antibodypositive polymyositis with cyclosporin. $\mathrm{Br} \mathrm{J}$ Rheumatol 1997; 36: $144-145$

90 Majmudar S, Hall HA, Zimmermann B. Treatment of adult inflammatory myositis with rituximab: an emerging therapy for refractory patients. Clin Rheumatol 2009; 15: 338-340

91 Mino M, Noma S, Taguchi Yet al. Pulmonary involvement in polymyositis and dermatomyositis: sequential evaluation with CT. AJR Am J Roentgenol 1997; 169: 83-87

92 Plotz PH, Rider LG, Targoff IN et al. NIH conference. Myositis: immunologic contributions to understanding cause, pathogenesis, and therapy. Ann intern Med 1995; 122: 715-724

93 Miller FW. Classification and prognosis of inflammatory muscle disease. Rheum Dis Clin N Amer 1994; 20: 811 -826

94 Fujisawa T, Suda T, Nakamura Y et al. Differences in clinical features and prognosis of interstitial lung diseases between polymyositis and dermatomyositis. J Rheumatol 2005; 32: 58-64
95 Haslam PL, Turton CW, Lukoszek A et al. Bronchoalveolar lavage fluid cell counts in cryptogenic fibrosing alveolitis and their relation to therapy. Thorax 1980; 35: 328-339

96 Zantos D, Zhang Y, Felson D. The overall and temporal association of cancer with polymyositis and dermatomyositis. J Rheumatol 1994; 21: $1855-1859$

97 Callen JP. Myositis and malignancy. Curr Opin Rheumatol 1994; 6: $590-594$

98 Vencovský J, Jarosová K, Machácek S et al. Cyclosporine A versus methotrexate in the treatment of polymyositis and dermatomyositis. Scand J Rheumatol 2000; 29: 95-102

99 Sem M, Molberg O, Lund MB, Gran JT. Rituximab treatment of the antisynthetase syndrome: a retrospective case series. Rheumatology 2009; 48: $968-971$

100 Hayashi S, Tanaka M, Kobayashi H et al. High-resolution computed tomography characterization of interstitial lung diseases in polymyositis/dermatomyositis. J Rheumatol 2008; 35: 260 - 269

101 Atzeni F, Doria A, Carrabba M et al. Potential target of infliximab in autoimmune and inflammatory diseases. Autoimmun Rev 2007; 6 : $529-536$

102 Suzuki Y, Hayakawa H, Miwa S et al. Intravenous immunoglobulin therapy for refractory interstitial lung disease associated with polymyositis/dermatomyositis. Lung 2009; 187: 201 - 206 